\title{
Pandemia da Covid 19: refletindo as vulnerabilidades a luz do gênero, raça e classe
}

\author{
Covid-19 Pandemic: reflecting vulnerabilities in the light of gender, \\ race and class
}

Fernanda Matheus Estrela (https://orcid.org/0000-0001-7501-6187) ${ }^{1}$

Caroline Fernandes Soares e Soares (https://orcid.org/0000-0003-4464-8389) ${ }^{1}$

Moniky Araújo da Cruz (http://orcid.org/0000-0003-2955-5408) ${ }^{2}$

Andrey Ferreira da Silva (http://orcid.org/0000-0002-1038-7443) ${ }^{2}$

Jemima Raquel Lopes Santos (https://orcid.org/0000-0002-9466-0936) ${ }^{2}$

Tânia Maria de Oliveira Moreira (https://orcid.org/0000-0002-4541-6750) ${ }^{1}$

Adriana Braitt Lima (https://orcid.org/0000-0001-7893-9753) ${ }^{1}$

Márcia Gomes Silva (https://orcid.org/0000-0001-5571-0649) ${ }^{1}$
${ }^{1}$ Departamento de Saúde, Universidade Estadual de Feira de Santana. Av. Transnordestina s/n, Novo Horizonte. 44036-900 Feira de Santana BA Brasil. nanmatheus@yahoo.com.br ${ }^{2}$ Universidade Federal da Bahia. Salvador BA Brasil.
Abstract This study aims to reflect the impact of COVID 19, considering gender, race, and class markers. This is an exploratory study, with an emphasis on the analysis of selected publications, based on a systematized search on official websites, and on the PubCovid-19 platform that includes papers published on COVID-19, which are indexed in PubMed and EMBASE. This work was based on these documents and built with reflections from the authors from the perspectives of social markers related to gender, race, and class, which contribute to the prognosis of the disease. The reflection carried out from the analyzed literature revealed that the markers of gender, class, and race emerge as a vulnerable condition to the exposure of COVID-19 in the most diverse world scenarios. This context reveals the historical need to implement strategies to improve the lives of this population, not only during the pandemic but also after their passing. Therefore, it is necessary to adopt socioeconomic policies with a more significant impact on the lives of these people and with greater coverage, expanding access to better health, education, housing, and income.

Key words COVID-19, Pandemic, Race and health, Gender, Social class
Resumo O objetivo deste estudo é refletir os impactos da COVID 19, considerando marcadores de gênero, raça e classe. Trata-se de um estudo exploratório, com ênfase na análise de publicações selecionados, a partir de procura sistematizada em sites oficiais, bem como na plataforma PubCovid-19 que apresenta os artigos publicados sobre COVID-19, os quais estão indexados nas Pubmed e EMBASE. O trabalho foi pautado nos referidos documentos e construido com reflexões dos autores a partir das perspectivas dos marcadores sociais relacionados à gênero, raça e classe, os quais contribuem para o prognóstico da doença. A reflexão realizada com base na literatura analisada revelou que os marcadores de gênero, classe e raça se apresentam enquanto condição vulnerabilizadora à exposição da COVID-19 nos mais diversos cenários mundiais. Esse contexto descortina a necessidade histórica da implantação de estratégias de melhoria de vida dessa população não só durante a pandemia, como também após sua passagem. Para tanto, necessário se faz a adoção de politicas socioeconômicas de maior impacto na vida dessas pessoas e com maior abrangência, ampliando o acesso a melhores condições de saúde, educação, moradia e renda.

Palavras-chave Covid-19, Pandemia, Raça e saúde, Gênero, Classe social 


\section{Introdução}

Desde o surgimento da COVID-19 até os dias atuais, a doença tem se propagado rapidamente no mundo inteiro. Sua expansão tem trazido impactos econômicos, sociais e para a saúde, principalmente quando se transversalizam os marcadores sociais de raça, classe e gênero, condições que vulnerabilizam diversos grupos populacionais. Dessa forma, sinaliza-se a necessidade de discussão acerca desses marcadores, de modo a compreender e superar os inúmeros desafios que permeiam esse cenário.

Com o advento da COVID-19, as repercussões nas áreas econômicas e de saúde foram os primeiros a serem percebidos. Estima-se o déficit de US\$ 1 trilhão na economia mundial durante o ano de 2020 decorrentes de gastos para saúde e com o sustento das famílias ${ }^{1}$. Para além disso, o advento da pandemia repercutiu de forma avassaladora na vida de pessoas que já se encontravam em situação de vulnerabilidade social por conta da informalidade do trabalho, má condição de moradia e dificuldade de acesso aos serviços de saúde².

O contexto desses públicos relaciona a convergência dos marcadores sociais que atuam distintamente, vulnerabilizando-os em suas individualidades. Além disso, a doença causada pelo novo Coronavírus, SARS-COV-2, COVID-19, apresenta, em sua maioria, sintomatologia leve, contudo, pode desenvolver quadros graves em grupos específicos, a exemplo das populações de rua. Esse público antes da pandemia já se encontrava em situação de vulnerabilidade, entretanto, a situação se agravou com a doença visto interrelação desta com os campos biológicos e sociais. Dessa forma, é possível identificar que os fatores que contribuem para a rápida disseminação do SARS-COV-2 estão atrelados não apenas às características de patogenicidade do vírus, mas também os determinantes sociais.

Frente a essas situações, pessoas têm sido atingidas, sobretudo aquelas com menores rendas e em condições sociais desfavoráveis, o que sinaliza para a reflexão dos marcadores que geram as desigualdades social. Considerando esse cenário e a realidade brasileira no que diz respeito às desigualdades, o objetivo deste estudo é refletir os impactos da COVID 19, considerando marcadores de gênero, raça e classe

\section{Metodologia}

Trata-se de um estudo exploratório, com ênfase na análise de publicações escritas e divulgadas por órgãos institucionais da rede pública de saúde e produções científicas referentes à pandemia da COVID 19. O trabalho foi pautado nos referidos documentos e construído com reflexões dos autores a partir das perspectivas dos marcadores sociais relacionados à gênero, raça e classe, os quais contribuem para o prognóstico da doença.

A reflexão é inerente ao pesquisador, uma vez que o conhecimento depende dela para ser construído. Essa afirmação é corroborada por Pierre Bourdieu $^{3}$, que destaca a importância da reflexividade na prática de pesquisa ao afirmar que esta postura representa não apenas o pensar, mas o agir sobre o tema e os indivíduos que são estudados. Deste modo, frente ao contexto atual de pandemia e à necessidade de constante construção de conhecimento que embase a prática e sirva de subsídio para olhar para públicos vulneráveis, a reflexão se constitui como método essencial no repensar e reorganizar o cuidado.

Os documentos utilizados foram selecionados, a partir de procura sistematizada em sites oficiais, bem como na plataforma PubCovid-19 que apresenta os artigos publicados sobre COVID-19, os quais estão indexados nas Pubmed e EMBASE. A plataforma, recém-criada, surgiu com o objetivo de compilar as publicações relacionadas à temática, uma vez que o conhecimento sobre a COVID-19 vem sendo publicado em alta velocidade. Nela há uma organização dos artigos por áreas temáticas a fim de facilitar o acesso e direcionar o pesquisador.

O critério de inclusão foram os artigos que versavam sobre a COVID-19 e tinham interface com gênero, raça e classe, incluindo a população de rua. Como critério de exclusão foi considerada a indisponibilidade do artigo na íntegra e de forma gratuita. A coleta de dados ocorreu no mês de abril de 2020 e foi associada a um instrumento que permitiu a coleta dos seguintes dados: nome dos autores, ano da publicação, idioma e identificação do recorte do marcador social.

O preenchimento desse instrumento deu origem aos quadros abaixo que sintetizam os achados (Quadro 1) e (Quadro 2). Os estudos foram lidos exaustivamente e analisados com vistas a busca dos elementos para o processo de discussão dos impactos dos marcadores de gênero, raça e classe na infecção por COVID-19. 
Quadro 1. Distribuição dos documentos selecionados conforme busca em sites oficiais.

\begin{tabular}{|c|c|c|}
\hline $\mathbf{N}^{\circ}$ & Título & Autores e ano de publicação \\
\hline 1 & $\begin{array}{l}\text { Prevenção ao COVID-19 no âmbito das equipes } \\
\text { de Consultório na Rua }{ }^{4}\end{array}$ & $\begin{array}{l}\text { Secretaria de Atenção Primária à Saúde (SAPS) } \\
2020\end{array}$ \\
\hline 2 & $\begin{array}{l}\text { NOTA TÉCNICA No 04/2020 GVIMS/GGTES/ } \\
\text { ANVISA }^{5}\end{array}$ & $\begin{array}{l}\text { Secretaria de Atenção Primária à Saúde (SAPS) } \\
2020\end{array}$ \\
\hline 3 & $\begin{array}{l}\text { Risco dos bairros de Salvador ao espalhamento } \\
\text { do COVID-19 decorrente da circulação de } \\
\text { pessoas e condições socioeconômicas }\end{array}$ & $\begin{array}{l}\text { Universidade Federal da Bahia (UFBA)- Grupo Geo } \\
\text { Combate COVID-19 BA } \\
2020\end{array}$ \\
\hline 4 & $\begin{array}{l}\text { Protocolo de Tratamento do Novo Coronavírus } \\
(2019-\mathrm{nCoV})^{7}\end{array}$ & $\begin{array}{l}\text { Ministério da Saúde } \\
2020\end{array}$ \\
\hline 5 & $\begin{array}{l}\text { Saúde da população em Situação de rua; um } \\
\text { direito humano }\end{array}$ & $\begin{array}{l}\text { Secretaria de Gestão Estratégica e Participativa } \\
2014\end{array}$ \\
\hline 6 & Desigualdades Sociais por Cor ou Raça no Brasil ${ }^{9}$ & $\begin{array}{l}\text { Instituto Brasileiro de Geografia e Estatísticas. (IBGE) } \\
2018\end{array}$ \\
\hline 7 & $\begin{array}{l}\text { Política Nacional para a População em Situação } \\
\text { de Rua }^{10}\end{array}$ & $\begin{array}{l}\text { Decreto } n^{\circ} 7.053 \text {, de } 23 \text { de dezembro de } \\
2009 .\end{array}$ \\
\hline
\end{tabular}

Quadro 2. Distribuição dos artigos selecionados conforme busca em plataforma PubCovid-19.

\begin{tabular}{|c|c|c|}
\hline $\mathrm{N}^{\circ}$ & Título & Autores e ano de publicação \\
\hline 1 & The Brazilian slums hiring their own doctors to fight covid- $19^{11}$ & $\begin{array}{l}\text { Andrade RO } \\
2020\end{array}$ \\
\hline 2 & $\begin{array}{l}\text { The vulnerability of low-and middle-income countries facing } \\
\text { the COVID-19 pandemic: The case of Haiti }{ }^{12}\end{array}$ & $\begin{array}{l}\text { Cénat JM } \\
2020\end{array}$ \\
\hline 3 & $\begin{array}{l}\text { Covid-19 is an opportunity for gender equality within the } \\
\text { workplace and at home }{ }^{13}\end{array}$ & $\begin{array}{l}\text { Wenham C et al. } \\
2020\end{array}$ \\
\hline 4 & $\begin{array}{l}\text { COVID-19: Vulnerability and the power of privilege in a } \\
\text { pandemic }^{14}\end{array}$ & $\begin{array}{l}\text { Smith JA, Judd J } \\
2020\end{array}$ \\
\hline 5 & $\begin{array}{l}\text { If the world fails to protect the economy, COVID-19 will } \\
\text { damage health not just now but also in the future }{ }^{15}\end{array}$ & $\begin{array}{l}\text { McKee M, Stuckler D } \\
2020\end{array}$ \\
\hline 6 & $\begin{array}{l}\text { Racial Capitalism: A Fundamental Cause of Novel Coronavirus } \\
(\text { COVID-19) })^{16}\end{array}$ & $\begin{array}{l}\text { Pirtle WNL } \\
2020\end{array}$ \\
\hline 7 & $\begin{array}{l}\text { Efforts escalate to protect homeless people from COVID-19 in } \\
\text { UK }^{17}\end{array}$ & $\begin{array}{l}\text { Kirby T } \\
2020\end{array}$ \\
\hline 8 & Racism and discrimination in COVID-19 responses ${ }^{18}$ & $\begin{array}{l}\text { Devakumar D et al. } \\
2020\end{array}$ \\
\hline 9 & $\begin{array}{l}\text { COVID-19 precautions: easier said than done when patients are } \\
\text { homeless }^{19}\end{array}$ & $\begin{array}{l}\text { Wood LJ et al. } \\
2020\end{array}$ \\
\hline
\end{tabular}

\section{Discussão}

Em tempos difíceis, face ao crescimento exponencial da COVID-19, é importante refletir quanto a vulnerabilidade de grupos específicos. Embora o vírus SARS-COV-2 não apresente seletividade contagiosa, os impactos da infecção serão sentidos de maneiras diferentes a depender da raça, classe e gênero. Tais marcadores, em razão das desigualdades produzidas socialmente, afetam as pessoas em diversas áreas das suas vidas para além da saúde.

No Brasil, os primeiros casos foram associados às classes economicamente favorecidas, devido às viagens internacionais, as quais se infectavam e, posteriormente, retornavam ao país. De acordo com o Ministério da Saúde ${ }^{20}$, o primeiro caso de brasileiro infectado foi notificado no dia 26 de fevereiro de 2020 em um paciente que havia retornado recentemente de uma viagem à Itália. 
Na periferia, os relatos foram de empregadas domésticas, motoristas de aplicativo, entregadores de alimentos que, em contato com aqueles que viajaram, se infectavam e levavam para suas casas. Assim, as pessoas de baixa renda, moradoras dos bairros periféricos de Salvador passaram a também estarem expostos à doença pelo fato de trabalharem nos locais mais nobres da cidade, onde há o maior percentual de pessoas infectadas pelo SARS-COV-2 ${ }^{6}$.

Cabe destacar que ser infectado se põe de igual forma para as pessoas, contudo, há diferenças nas medidas de prevenção e possibilidade de agravamento dos sinais e sintomas. No que tange à classe, observa-se que o baixo nível de escolaridade associado à pobreza extrema tem impacto direto no descumprimento às instruções de saúde pública. Essa situação é desafiadora em todo o mundo e vem sendo apontada principalmente em países emergentes. No Haiti, com uma população de 11 milhões de habitantes, menos de 30 leitos de UTI e ausência de equipamentos de proteção aos prestadores dos serviços, a falta de recursos é ainda mais expressiva frente a não adoção de medidas preventivas, gerando uma grave crise na saúde ${ }^{12}$.

Tendo em vista esse cenário, o efeito adverso na saúde produz um declínio econômico dos indivíduos e famílias, uma vez que o controle da doença requer estratégias como distanciamento social e quarentena. Tais medidas se aplicam com impactos nas populações de baixa renda que têm pouco acesso aos cuidados de saúde e necessitam escolher entre ficar em casa e passar fome ou correr os riscos do descumprimento ao isolamento para o sustento de si e da família ${ }^{15}$.

No que tange ao campo de trabalho, além daqueles que não podem atender às medidas restritivas por dependerem economicamente desses proventos para sobreviverem, há outros que desempenham atividades essenciais e, por isso, estão expostos ${ }^{6}$. Um exemplo desse público são os homens com altas taxas de incidência da doença, o que pode guardar relação com a provisão de seus lares. Pouco se discute quanto ao impacto de gênero frente ao surto do Covid-19, na qual observamos uma posição de neutralidade das políticas públicas, como se homens e mulheres fossem infectados e afetados igualmente.

No Brasil, recortes de classe também estão ligados à raça, uma vez que segundo dados divulgados pelo Instituto Brasileiro de Geografia e Estatística, em 2018, pela pesquisa "Desigualdades Sociais por Cor ou Raça no Brasil”, 75\% das pessoas que vivem na extrema pobreza são autode- clarados como negros ou pardos 9 . As iniquidades sociais dos países organizam suas sociedades de maneira a torná-las extremamente vulneráveis. Dessa forma, os desfavorecidos socioeconomicamente são representados por minorias raciais e étnicas que trabalham em empregos casuais e carecem de recursos financeiros necessários para o autoisolamento. Em contrapartida, uma elite cada vez mais pequena e seleta demonstra o poder do privilégio em uma pandemia, na qual os mais vulneráveis serão os mais atingidos ${ }^{14,15,18}$.

Ainda tratando da raça como marcador social, o capitalismo racial constitui uma causa fundamental de iniquidades em saúde. Um estudo desenvolvido na cidade Detroit do EUA com apenas $14 \%$ de sua população sendo negra, mostrou que $40 \%$ da mortalidade por COVID-19 são de negros ${ }^{16}$. Isso pode guardar relação com as altas taxas de comorbidades nessa população que as vulnerabiliza para o agravamento do quadro da COVID-19. Relatório com dados da Itália mostrou associação entre quadros graves da doença e diabetes, situação que é incidente na população negra do Sul da Ásia, conforme pesquisa ${ }^{18}$. Deste modo, a raça e os aspectos que a tranversalizam são elementos vulnerabilizadores para a COVID-19.

Essas diferenças são evidências de um racismo estrutural que dificulta a vida de negros e negras desde o acesso a testes para detecção do vírus até o tratamento da infecção, facilitado por poder, dinheiro e prestígios que podem aliviar as consequências da doença. Estudo revela que Detroit está entre as 20 principais cidades dos Estados Unidos com os maiores números de pessoas em situação de rua e a maioria destas pessoas são negras ${ }^{16}$. O desabrigo, os mais pobres e os negros estão claramente mais vulneráveis às consequências do COVID-19.

Viver em situação de rua expõe a inúmeras situações adoecedoras, sendo mais latente na pandemia. A dificuldade de acesso aos serviços de saúde, somado ao preconceito, reflete em impactos ainda maiores. Observa-que quando pacientes se apresentam nos departamentos de emergência e em hospitais com sintomas de COVID-19, suas queixas podem ser minimizadas, o que ratifica a dificuldade na manutenção da saúde. Esta população é muitas vezes excluída socialmente, o que acabam por cercear uma gama de direitos concernentes ao ser humano. Esta invisibilidade é percebida nas políticas públicas, visto que, somente em 2009, com do Decreto no 7.053 , de 23 de dezembro de 2009 foi instituída a Política Nacional para a População em Situação de 
Rua. A mesma, considera pessoas em situação de rua aquelas que atendem aos seguintes requisitos: estar em condição de extrema pobreza, ter vínculos familiares interrompidos ou fragilizados, não ter moradia convencional regular e utilizar ruas públicas e/ou áreas degradadas para abrigar-se ou sustentar-se ${ }^{10}$.

Em todo o Reino Unido, uma grande equipe está trabalhando a uma velocidade vertiginosa para implantar sistemas para proteger as pessoas em situação de rua dos efeitos potencialmente devastadores causados pela doença por coronavírus 2019 (COVID-19). Esse grupo geralmente tem uma prevalência muito maior de condições crônicas como doenças pulmonares, diabetes e doenças cardiovasculares em comparação com pessoas da mesma idade que estão alojadas ${ }^{17,19}$. Além disso, compartilham acomodações apertadas, em aglomerações, sendo portanto, vulneráveis a altas taxas de infecção e sintomatologia grave ${ }^{17}$. Como cumprir com o isolamento social e se abrigarem em um local que não tem abrigo? Se não há água para beber e água para tomar banho, lavar as mãos com água e sabão não tornou-se um privilégio? Por mais que a recomendação seja não compartilhar objetos pessoais, o menos importante durante a fome e o frio é saber de onde veio aquela comida desperdiçada ou aquela roupa doada.

No Brasil, o município de Salvador, através da Nota Técnica No 04/2020, orientou sobre a forma de facilitar o acesso das pessoas em situação de rua aos cuidados de saúde frente à pandemia do COVID-19. Esta norma orienta a respeito do acolhimento, da definição do caso, notificação, diagnóstico, manejo clínico, acompanhamento em Unidade de Acolhimento ou domiciliar, ações sobre o Consultório de Rua e medidas de prevenção e controle 5 .

Em caso de haver a necessidade de isolamento social, a equipe que acolheu este paciente fica responsável pela identificação de um possível domić́lio no qual possa repousar. Também deve obter contato telefônico e endereço, a fim de garantir o seguimento do tratamento/cuidado. Caso não exista um domicílio é necessário que haja um contato com o serviço de Abordagem Social, a fim de regular o caso para o auxílio moradia ${ }^{4}$.

Essa população vulnerabilizada e marginalizada, é resultado de um fenômeno social, tendo como moradia os logradouros, pontes e/ou albergues, contudo este não é o único desafio vivenciado por estas pessoas, pois ainda vivem sem acesso aos serviços básicos de Saúde. Urge que sejam criadas estratégias em todo o mundo para aumentar o acesso a saúde a essa população.

\section{Considerações finais}

A reflexão realizada com base na literatura analisada revelou que os marcadores de gênero, classe e raça se apresentam enquanto condição vulnerabilizadora à exposição da COVID-19 nos mais diversos cenários mundiais. Esse contexto descortina a necessidade histórica da implantação de estratégias de melhoria de vida dessa população não só durante a pandemia, como também após sua passagem. Para tanto, necessário se faz a adoção de políticas socioeconômicas de maior impacto na vida dessas pessoas e com maior abrangência, ampliando o acesso a melhores condições de saúde, educação, moradia e renda.

\section{Colaboradores}

FM Estrela, CFS Soares, MA Cruz, AF Silva e JRL Santos contribuíram com a concepção e projeto, redação do artigo e aprovação final da versão a ser publicada. TMO Moreira, AB Lima e MG Silva contribuíram com a concepção e projeto, revisão crítica do artigo e aprovação final da versão a ser publicada. 


\section{Referências}

1. Kabir M, Afzal MS, Khan A, Ahmed H. COVID-19 pandemic and economic cost; impact on forcibly displaced people. Travel Med Infect Dis [Internet]. 2020 Apr [cited 2020 May 1];101661. Available from: https://linkinghub.elsevier.com/retrieve/pii/S1477 893920301290

2. Instituto Brasileiro de Geografia e Estatísticas (IBGE). Pesquisa Nacional por Amostra de Domicílios [Internet]. Rio de Janeiro: IBGE; 2019. [cited 2020 Apr 30]. Available from: https://agenciadenoticias.ibge.gov.br/ media/com_mediaibge/arquivos/8ff41004968ad3630 6430c82eece3173.pdf

3. Bourdieu P. O poder simbólico. Rio de Janeiro: Bertrand Brasil; 2005.

4. Brasil. Ministério da Saúde (MS). Prevenção ao covid-19 no âmbito das equipes de consultórios na rua [Internet]. Brasília: Secretaria de atenção primária à saúde; 2020. [cited 2020 Apr 14]. Available from: http://189.28.128.100/dab/docs/portaldab/documentos/Consultorios_rua_APS_20200319_ver001.pdf

5. Brasil. Ministério da Saúde (MS). Nota técnica GVIMS/GGTES/anvisa no 04/2020 [Internet]. $4^{\text {a }}$ ed. Brasília: Secretaria de atenção primária à saúde; 2020. [cited 2020 Apr 14]. Available from: https://saude. rs.gov.br/upload/arquivos/202004/01082621-nota-tecnica-n-04-2020-gvims-ggtes-anvisa-atualizada-3.pdf

6. Universidade Federal da Bahia (UFBA). Grupo GeoCombate COVID-19 BA. Risco dos bairros de Salvador ao espalhamento do COVID-19 decorrente da circulação de pessoas e condições socioeconômicas. Salvador: UFBA; 2020. [cited 2020 Apr 14]. Available from: http://www.edgardigital.ufba.br/?p=16356

7. Brasil. Ministério da Saúde (MS). Protocolo de Tratamento do Novo Coronavírus (2019-nCoV). Brasília: MS; 2020. [cited 2020 Apr 10]. Available from: https:// portalarquivos2.saude.gov.br/images/pdf/2020/fevereiro/05/Protocolo-de-manejo-clinico-para-o-novocoronavirus-2019-ncov.pdf

8. Brasil. Ministério da Saúde (MS). Saúde da população em situação de rua: um direito humano. Brasília: MS; 2014 [cited 2020 Apr 2018]. Available from: https:// bvsms.saude.gov.br/bvs/publicacoes/saude_populacao_situacao_rua.pdf

9. Instituto Brasileiro de Geografia e estatística (IBGE). Desigualdades Sociais por Cor ou Raça no Brasil. 41 ${ }^{\mathrm{a}}$ ed. Brasília: Estudos e Pesquisas: Informação Demográfica e Socioeconômica; 2018. [cited 2020 Apr 19]. Available from: https://biblioteca.ibge.gov.br/visualizacao/livros/liv101681_informativo.pdf

10. Brasil. Decreto no 7.053, de 23 de dezembro de 2009. Institui a Política Nacional para a População em Situação de Rua e seu Comitê Intersetorial de Acompanhamento e Monitoramento, e dá outras providências. Diário Oficial da União 2009; 24 dez.

11. Andrade RA. The Brazilian slums hiring their own doctors to fight covid-19. BMJ [Internet]. $2020 \mathrm{Apr} 22$ [cited 2020 May 1];369:m1597. Available from: http:// www.bmj.com/lookup/doi/10.1136/bmj.m1597
12. Cénat JM. The vulnerability of low-and middle-income countries facing the COVID-19 pandemic: The case of Haiti. Travel Med Infect Dis [Internet]. 2020 Apr [cited 2020 Apr 25];101684. Available from: https://linkinghub.elsevier.com/retrieve/pii/S1477 893920301526

13. Wenham C, Smith J, Morgan R. Covid-19 is an opportunity for gender equality within the workplace and at home. BMJ [Internet]. 2020 Apr 20 [cited 2020 May 1]; 369: m1546. Available from: http://www.bmj.com/ lookup/doi/10.1136/bmj.m1546

14. Smith JA, Judd J. COVID-19: Vulnerability and the power of privilege in a pandemic. Heal Promot J Aust [Internet]. 2020 Apr [cited 2020 May 1]; 31(2):158160. Available from: https://onlinelibrary.wiley.com/ doi/abs/10.1002/hpja.333

15. McKee M, Stuckler D. If the world fails to protect the economy, COVID-19 will damage health not just now but also in the future. Nat Med [Internet]. 2020 Apr 9 [cited 2020 May 1]; Available from: http://www.nature.com/articles/s41591-020-0863-y

16. Pirtle WNL. Racial Capitalism: A Fundamental Cause of Novel Coronavirus (COVID-19) Pandemic Inequities in the United States. Heal Educ Behav [Internet]. 2020 Apr 26 [cited 2020 May 1];109019812092294. Available from: http://journals.sagepub.com/doi/ $10.1177 / 1090198120922942$

17. Kirby T. Efforts escalate to protect homeless people from COVID-19 in UK. Lancet Respir Med [Internet]. 2020 Mar [cited 2020 May 1]; Available from: https://linkinghub.elsevier.com/retrieve/pii/S2213 260020301600

18. Devakumar D, Shannon G, Bhopal SS, Abubakar I. Racism and discrimination in COVID-19 responses. Lancet 2020; 395(10231):1194.

19. Wood LJ, Davies AP, Khan Z. COVID-19 precautions: easier said than done when patients are homeless. Med J Aust [Internet]. 2020 Apr 8 [cited 2020 May 1]; Available from: http://doi.wiley.com/10.5694/mja2.50571

20. Brasil. Ministério da Saúde (MS). Brasil confirma primeiro caso da doença. Brasília: MS; 2020 [cited 2020 May 1]. Available from: https://www.saude.gov.br/ noticias/agencia-saude/46435-brasil-confirma-primeiro-caso-de-novo-coronavirus

Artigo apresentado em 01/05/2020

Aprovado em 14/05/2020

Versão final apresentada em 16/05/2020 